\title{
MANIFOLDS HOMEOMORPHIC TO SPHERE BUNDLES OVER SPHERES
}

\author{
BY R. DE SAPIO ${ }^{1}$
}

\author{
Communicated by William Browder, July 9, 1968
}

1. Statement of results. Let $E$ be the total space of a $k$-sphere bundle over the $n$-sphere with characteristic class $\alpha \in \pi_{n-1}\left(S O_{k+1}\right)$. We consider the problem of classifying, under the relation of orientation preserving diffeomorphism, all differential structures on $E$. It is assumed that $E$ is simply connected, of dimension greater than five, and its characteristic class $\alpha$ may be pulled back to lie in $\pi_{n-1}\left(S_{k}\right)$ (that is, the bundle has a cross-section). In [1] and [2] we gave a complete classification in the special case where $\alpha=0$. The more general classification Theorems 1 and 2 below include this special case. The proofs of these theorems are sketched in $\$ 2$ below; detailed proofs will appear elsewhere. J. Munkres [6] has announced a classification up to concordance of differential structures in the case where the bundle has at least two cross-sections. (It is well known that concordance and diffeomorphism are not equivalent, concordance of differential structures being strictly stronger than diffeomorphism.)

ThEOREM 1. Let $E$ be the total space of a $k$-sphere bundle over the $n$-sphere whose characteristic class ${ }^{2} \alpha$ may be pulled back to lie in $\pi_{n-1}\left(S O_{k}\right)$. Suppose that $2 \leqq k<n-1$. Then, under the relation of orientation preserving diffeomorphism, the diffeomorphism classes of manifolds homeomorphic to $E$ are in a one-to-one correspondence with the equivalence classes on the set $\left(\theta_{n} / \Phi_{n}^{k+1}\right) \times \theta_{n+k}$, where $\left(A_{*}^{n}, U^{n+k}\right)$ and $\left(B_{*}^{n}, V^{n+k}\right)$ are equivalent if and only if $A_{*}^{n}= \pm B_{*}^{n}$ and there exists $\beta \in \pi_{k}\left(S O_{n-1}\right)$ such that $U^{n+k}-V^{n+k}=\tau_{n, k}^{\prime}\left(A_{*}^{n} \otimes \beta\right)+\sigma_{n-1, k}(\alpha \otimes \beta)$.

Theorem 1 is also true in the case where $k=n-1$ and $n$ is odd. The classification in the case where $n-1 \leqq k \leqq n+2$ is essentially the same as the above and is given in Theorem 2 below. Now we establish the notation used in Theorem 1.

Notation. Manifolds and diffeomorphisms are of class $C^{\infty}$. The group of homotopy $n$-spheres under the connected sum operation +

\footnotetext{
1 The preparation of this paper was supported in part by National Science Foundation Grant \# GP 7036.

2 Added in proof. Assume here and in Proposition 2 that $\alpha$ is of order 2 in $\pi_{n-1}$ $\left(\mathrm{SO}_{k+1}\right)$ in the case where $k<n-3$. This assumption is not made elsewhere.
} 
is denoted by $\theta_{n}$, and $\Phi_{n}^{k+1}$ is the subgroup of $\theta_{n}$ consisting of those homotopy $n$-spheres that embed in $(n+k+1)$-space with a trivial normal bundle. The class of a homotopy $n$-sphere $A^{n}$ in the group $\theta_{n} / \Phi_{n}^{k+1}$ is denoted by $A_{*}^{n}$. Now let

$$
\begin{aligned}
\sigma_{n-1, k}: \pi_{n-1}\left(S O_{k}\right) \otimes \pi_{k}\left(S O_{n-1}\right) & \rightarrow \theta_{n+k}, \\
\tau_{n, k}: \theta_{n} \otimes \pi_{k}\left(S O_{n-1}\right) & \rightarrow \theta_{n+k}
\end{aligned}
$$

be the pairings defined in $[4$, p. 583]. It is known that these pairings correspond to composition in the stable homotopy groups of spheres. Moreover, it was shown in [2] that $\tau_{n, k}\left(\Phi_{n}^{k+1} \otimes \pi_{k}\left(S O_{n-1}\right)\right)=0$, provided that $k \geqq 2$, and hence the pairing $\tau_{n, k}$ induces a pairing

$$
\tau_{n, k}^{\prime}:\left(\theta_{n} / \Phi_{n}^{k+1}\right) \otimes \pi_{k}\left(S O_{n-1}\right) \rightarrow \theta_{n+k} \quad(k \geqq 2) .
$$

REMARK. If $k \geqq n-3$, then $\Phi_{n}^{k+1}=\theta_{n}$ (see [1, Lemma 1]) and hence $\tau_{n, k}=\tau_{n, k}^{\prime}=0$ for $k \geqq n-3$.

In order to state the result in the case where $n-1 \leqq k \leqq n+2$ we define a function

$$
\sigma_{n, k}^{\prime}: \pi_{n-1}\left(S O_{k}\right) \times \pi_{k}\left(S O_{n}\right) \rightarrow \theta_{n+k}
$$

that is linear in the second variable. The definition of $\sigma_{n, k}^{\prime}$ is similar to the definition of the pairing $\sigma_{n-1, k}$ and is described in $\$ 2$ below. Now if $\alpha \in \pi_{n-1}\left(S O_{k}\right)$ is the characteristic class of the bundle $E$, then we define a homomorphism

$$
\chi_{\alpha}: \pi_{k}\left(S O_{n}\right) \rightarrow \theta_{n+k}
$$

by writing, for each $\beta \in \pi_{k}\left(S O_{n}\right)$,

$$
\chi_{\alpha}(\beta)=\sigma_{n, k}^{\prime}(\alpha, \beta) .
$$

TheOREM 2. Suppose that the characteristic class $\alpha$ of the bundle $E$ may be pulled back to lie in $\pi_{n-1}\left(S O_{k}\right)$. Then, if $1 \leqq n-3 \leqq k \leqq n+2$ and $k \geqq 2$, then the diffeomorphism classes of manifolds homeomorphic to $E$ are in a one-to-one correspondence with the group $\theta_{n+k} /$ Image $\chi_{\alpha}$.

2. Outline of proofs. We give $E$ the "standard" differential structure by making it a smooth $k$-sphere bundle over the standard $n$ sphere $S^{n}$. It is well known that if a $k$-sphere bundle over the $n$-sphere has a cross-section, then the total space of the bundle has the homology of the product $S^{n} \times S^{k}$. The proof of Theorem 1 is divided into the following four propositions. We use the notation $E\left(A^{n}\right)$ to denote the differential $(n+k)$-manifold obtained by making $E$ into a smooth $k$-sphere bundle over a homotopy $n$-sphere $A^{n}$ in the obvious way (if 
$n=4$, then take $A^{4}$ to be homeomorphic to $\left.S^{4}\right)$. We assume that $E$ has a cross-section, $n>3$, and $n+k>5$. We also assume that $k \geqq 2$, except in Proposition 4 where we allow $k=1$.

Proposition 1. If $M$ is a differential $(n+k)$-manifold that is homeomorphic to $E$, then there are homotopy spheres $A^{n}$ and $U^{n+k}$ such that $M$ is diffeomorphic to $E\left(A^{n}\right)+U^{n+k}$, provided that $k \leqq n+2$.

Sketch of Proof. Since $E$ is of dimension greater than five and simply connected we can apply the Hauptvermutung of [7] to conclude that there is a PL-homeomorphism $h: M \rightarrow E$, where the combinatorial structures are compatible with the differential structures. We try to smooth $h$ by applying the obstruction theory of Munkres [5]. If $k<n$, then the first obstruction to deforming $h$ into a diffeomorphism is an element $c(h)$ in $H_{n}\left(M ; \Gamma_{k}\right)$, where $\Gamma_{k}$ is the group of diffeomorphisms of $S^{k-1}$ modulo those that extend to diffeomorphisms of the $k$-disk $D^{k}$. Since $H_{n}\left(M ; \Gamma_{k}\right)$ is isomorphic to $\Gamma_{k}$ we can consider $c(h)$ to be an element of $\Gamma_{k}$. Now we construct a manifold $M(c(h))$ and a PL-homeomorphism $j$ from $E$ to $M(c(h))$ such that the first obstruction to smoothing $j$ is $-c(h)$. It follows that the first obstruction to smoothing the composition $j h$ is zero and hence we can suppose that $j h$ is a diffeomorphism modulo the $k$-skeleton. The next step is to show that there is a diffeomorphism modulo a point $\phi: M(c(h)) \rightarrow E$, (this is true for $k \leqq n+2)$ and hence the composition $h^{\prime}=\phi j h$ is a diffeomorphism modulo the $k$-skeleton. The first obstruction to smoothing $h^{\prime}: M \rightarrow E$ is an element $c\left(h^{\prime}\right)$ in $H_{k}\left(M ; \Gamma_{n}\right) \approx \Gamma_{n}$. Now let $A^{n}$ be the homotopy $n$-sphere that corresponds to $c\left(h^{\prime}\right)$ under the isomorphism $\Gamma_{n} \approx \theta_{n}(n \neq 3)$. There is a PL-homeomorphism $j^{\prime}$ from $E$ to $E\left(A^{n}\right)$. Moreover, the first obstruction to smoothing $j^{\prime}$ is $-c\left(h^{\prime}\right)$ and hence we can assume that the composition $j^{\prime} h^{\prime}$ is a diffeomorphism up to a point. It follows that there is a homotopy $(n+k)$-sphere $U^{n+k}$ such that $M$ is diffeomorphic to $E\left(A^{n}\right)+U^{n+k}$. The argument in the case where $n \leqq k \leqq n+2$ is essentially the same. Note that if $n=4$, then the homotopy sphere $A^{4}$ is homeomorphic and hence diffeomorphic to $S^{4}$ since $\Gamma_{4}=0$.

The remaining propositions combine to give a classification of manifolds of the form $E\left(A^{n}\right)+U^{n+k}$.

Proposition 2. $E\left(A^{n}\right)$ and $E\left(B^{n}\right)$ are diffeomorphic if and only if $A^{n} \equiv \pm B^{n} \bmod \Phi_{n}^{k+1}$.

The proof of Proposition 2 is similar to the proofs of Lemmas 5 and 6 of [1]. R. Schultz informs me that he has also proved Proposition 1 and Proposition 2. 
Proposition 3. If $E\left(A^{n}\right)+U^{n+k}$ is diffeomorphic to $E\left(B^{n}\right)$, where $A^{n}, B^{n}, U^{n+k}$ are homotopy spheres, then $E\left(A^{n}\right)$ and $E\left(B^{n}\right)$ are diffeomorphic.

The proof of Proposition 3 is similar to the proof of Lemma 3 of [1].

Proposition 4. Let $A^{n}, U^{n+k}$ be homotopy spheres such that $1 \leqq k$ $<n-1$. Then, $E\left(A^{n}\right)+U^{n+k}$ is diffeomorphic to $E\left(A^{n}\right)$ if and only if there exists an element $\beta \in \pi_{k}\left(S O_{n-1}\right)$ such that

$$
U^{n+k}=\tau_{n, k}\left(A^{n} \otimes \beta\right)+\sigma_{n-1, k}(\alpha \otimes \beta) .
$$

The proof of Proposition 4 is similar to the proof of Theorem 3.1 of [2] except that the proof here is a bit more complicated since there are two pairings involved rather than just the pairing $\tau_{n, k}$.

Now we give the construction of the function $\sigma_{n, k}^{\prime}$ of (1) in $\$ 1$. Let $\gamma: S^{n-1} \rightarrow S O_{k}$ and $\beta: S^{k} \rightarrow S O_{n}$ be differentiable maps that represent elements in $\pi_{n-1}\left(S O_{k}\right)$ and $\pi_{k}\left(S O_{n}\right)$, respectively. We can assume that $\beta$ maps the southern hemisphere $D_{-}^{\boldsymbol{k}}$ of $S^{k}$ into the identity of $S O_{n}$. Define diffeomorphisms $\lambda_{\gamma}$ and $\mu_{\beta}$ of $S^{n-1} \times S^{k}$ by writing, for each $(u, v) \in S^{n-1} \times S^{k}, \lambda_{\gamma}(u, v)=(u, s \gamma(u) \cdot v)$ and $\mu_{\beta}(u, v)=(\beta(v) \cdot u, v)$; here the dot denotes the action of the rotation group on the sphere and $s$ denotes the natural inclusion of $S O_{k}$ in $S O_{k+1}$. It is clear that $\lambda_{\gamma}\left(S^{n-1} \times D_{-}^{k}\right)=S^{n-1} \times D_{-}^{k}$ and hence it follows that the diffeomorphism $\lambda_{\gamma}^{-1} \mu_{\beta} \lambda_{\gamma}$ of $S^{n-1} \times S^{k}$ is the identity on $S^{n-1} \times D_{-}^{k}$. Now if $B^{n+k}$ is an $(n+k)$-disk in the interior of $D^{n} \times S^{k}$, then it follows that the diffeomorphism $\lambda_{\gamma}^{-1} \mu_{\beta} \lambda_{\gamma}$ can be extended to a diffeomorphism of $D^{n} \times S^{k}$ - Interior $B^{n+k}$. The diffeomorphism induced on the $(n+k-1)$-sphere $\partial B^{n+k}$ determines an element $\sigma_{n, k}^{\prime}(\gamma, \beta)$ of $\theta_{n+k}$, and it is not hard to show that this element depends only on the homotopy classes of $\gamma$ and $\beta$. In fact, $\sigma_{n, k}^{\prime}(\gamma, \beta)$ is the obstruction to extending $\lambda_{\gamma}^{-1} \mu_{\beta} \lambda_{\gamma}$ to a diffeomorphism of $D^{n} \times S^{k}$. Since obstructions are additive with respect to compositions and

$$
\lambda_{\gamma}^{-1} \mu_{\beta+\beta^{\prime}} \lambda_{\gamma}=\left(\lambda_{\gamma}^{-1} \mu_{\beta} \lambda_{\gamma}\right)\left(\lambda_{\gamma}^{-1} \mu_{\beta^{\prime}} \lambda_{\gamma}\right),
$$

the correspondence $(\gamma, \beta) \rightarrow \sigma_{n, k}^{\prime}(\gamma, \beta)$ is linear in $\beta$.

Proposition 5. Let $A^{n}$ and $U^{n+k}$ be homotopy spheres such that $1 \leqq n-3 \leqq k<2 n-3$. Then, $E\left(A^{n}\right)+U^{n+k}$ is diffeomorphic to $E\left(A^{n}\right)$ if and only if there exists an element $\beta \in \pi_{k}\left(S O_{n}\right)$ such that $U^{n+k}=\chi_{\alpha}(\beta)$.

Now Theorem 2 follows by applying Propositions 1, 2, and 5, noting that $\Phi_{n}^{k+1}=\theta_{n}$ for $k \geqq n-3$.

We conclude with some remarks on the case where $k>n+2$. Proposition 1 is not true in this case. For example let $\Sigma^{16}$ denote the non- 
zero element of $\theta_{16} \approx Z_{2}$. It is known that $\Sigma^{16}$ does not embed in $R^{29}$ with a trivial normal bundle [3, Theorem 1.3]. Suppose that the conclusion of Proposition 1 is true for $S^{12} \times \Sigma^{16}$; that is, suppose that $S^{12} \times \Sigma^{16}$ is diffeomorphic to $\left(A^{12} \times S^{16}\right)+U^{28}$ for homotopy spheres $A^{12}$ and $U^{28}$. It is well known that $A^{12} \times S^{16}$ is diffeomorphic to $S^{12} \times S^{16}$ and hence it follows that $S^{12} \times \Sigma^{16}$ and $S^{12} \times S^{16}$ are diffeomorphic up to a point. This implies that $\Sigma^{16}$ embeds in $R^{29}$ with a trivial normal bundle, a contradiction. On the other hand if $k>n+2$, then the characteristic class $\alpha$ may be pulled back to lie in $\pi_{n-1}\left(\mathrm{SO}_{k-2}\right)$. Define homomorphisms $\eta_{\alpha}: \theta_{k} \rightarrow \theta_{n+k-1}$ and $\eta_{\alpha}^{\prime}: \theta_{k+1} \rightarrow \theta_{n+k}$ by writing

$$
\eta_{\alpha}\left(\Sigma^{k}\right)=\tau_{k, n-1}\left(\Sigma^{k} \otimes \alpha\right) \text { and } \eta_{\alpha}^{\prime}\left(\Sigma^{k+1}\right)=\tau_{k+1, n-1}\left(\Sigma^{k+1} \otimes \alpha\right)
$$

for $\Sigma^{k} \in \theta_{k}$ and $\Sigma^{k+1} \in \theta_{k+1}$, respectively. It follows from [2] that $\Phi_{k}^{n} \subset$ Kernel $\eta_{\alpha}$. Moreover, we can show that the number of distinct (nondiffeomorphic) differential structures on $E$ is not greater than the order of Kernel $\eta_{\alpha} / \Phi_{k}^{n}$ times the order of $\theta_{n+k} /$ Image $\eta_{\alpha}^{\prime}$. We plan to give the explicit computation at a later date. Finally, it follows from Munkres [6] that the concordance classes of differential structures on $E$ are in a one-to-one correspondence with

$$
\theta_{n} \oplus\left(\text { Kernel } \eta_{\alpha}\right) \oplus\left(\theta_{n+k} / \text { Image } \eta_{\alpha}^{\prime}\right) \text {. }
$$

\section{REFERENCES}

1. R. De Sapio, Differential structures on a product of spheres, Comment. Math. Helv. (to appear).

2. - Differential structures on a product of spheres. II, Ann. of Math. (to appear).

3. W. C. Hsiang, J. Levine and R. H. Szczarba, On the normal bundle of a homotopy sphere embedded in Euclidean space, Topology 3 (1965), 173-181.

4. R. Lashof, Problems in differential and algebraic topology, Seattle Conference, 1963, Ann. of Math. (2) 81 (1965), 565-591.

5. J. Munkres, Obs!ructions to the smoothing of piecewise differentiable homeomorphisms, Ann. of Math. (2) 72 (1960), 521-554.

6. - Concordance of differentiable structures-two approaches, Michigan Math. J. 14 (1967), 183-191.

7. D. Sullivan, On the Hauptvermutung for manifolds, Bull. Amer. Math. Soc. 73 (1967), 598-600.

University of California, Los Angeles, California 90024 